\title{
A profile of vancomycin-resistant enterococcal infections and a comparison of resistance detection methods
}

\author{
Stephen K. Mathew \\ Assistant Professor, Dept. of Microbiology, Believers Church Medical College, Kuttapuzha, Thiruvalla, Kerala, India
}

*Corresponding Author:

Email: stephenmathew@live.com

Received: $17^{\text {th }}$ June, 2018

Accepted: $13^{\text {th }}$ July, 2018

\begin{abstract}
Scope: Vancomycin-resistant enterococci (VRE) are being increasingly reported from hospitals across the world. This study provides a profile of enterococcal infections and compares various methods of detecting vancomycin resistance.

Materials and Methods: All clinically significant isolates of enterococci over a 2-year period were included. Antibiotic susceptibility was carried out as per CLSI guidelines. Vancomycin resistance was detected by 3 methods: disk diffusion, agar screen, E-test. The 3 methods were compared.

Results: 156 clinical samples yielded Enterococcus spp. over the study period. Maximum resistance was noted to penicillin, erythromycin, and ciprofloxacin. E. faecium strains showed a higher percentage of resistance to the antibiotics tested. $15(9.6 \%)$ enterococcal strains were resistant to vancomycin; 10 (6.4\%) strains were intermediate. Compared to E-test, disk diffusion and agar screen had sensitivities of $100 \%$. Disk diffusion had $97.2 \%$ specificity and agar screen demonstrated $92.9 \%$ specificity.

Conclusion: Prevalence of VRE in Indian hospitals is increasing. Though disk diffusion had a higher specificity than the agar screen at identifying resistant isolates, intermediate strains were identified as sensitive. BHI agar containing $6 \mu \mathrm{g} / \mathrm{ml}$ of vancomycin can be used to screen for VRE, and E-test can be used to confirm resistance.
\end{abstract}

Keywords: Bacterial infections, Enterococcus, Drug resistance, Vancomycin, Vancomycin resistance.

\section{Introduction}

Resistance is a paradoxical, yet natural outcome of antibiotic use. Alexander Fleming, in 1945, warned that misuse of penicillin would lead to resistant organisms. ${ }^{1}$ The use of broad-spectrum antibiotic agents as a substitute for precise diagnostics increases the rate of selection of resistant bacteria.

Arguably, the most impressive accomplishment of bacteria in this arena has been the development of vancomycin resistance enterococci (VRE). ${ }^{2}$ Vancomycin had been in clinical use for more than thirty years when, in England in 1988, Uttley et al. first reported the isolation of VRE. . $^{3,4}$

Enterococci are Gram-positive cocci that are part of the normal gut flora. Among them, Enterococcus faecalis and Enterococcus faecium are predominantly isolated from clinical samples. Less commonly isolated species include E. gallinarum, E. casseliflavus, E. durans, E. avium and E. raffinosus. ${ }^{5}$ Infections caused by enterococci include urinary tract infections (UTIs), bacteremia, intra-abdominal infections, endocarditis, central nervous system (CNS) infections, skin and soft tissue infections. ${ }^{5-8}$

Enterococci exhibit intrinsic resistance to cephalosporins, low levels of aminoglycosides and clindamycin; acquired resistance is seen against highlevel aminoglycosides (HLAR), vancomycin, chloramphenicol, erythromycin, high levels of clindamycin, tetracycline, and fluoroquinolones. ${ }^{9}$

Glycopeptide-resistant enterococci have become a major threat to hospitalized patients, causing outbreaks that increase morbidity, mortality, and healthcareassociated costs. ${ }^{10}$

Currently, there are nine types of operon structure conferring glycopeptide resistance, designated according to the characteristics of a key ligase gene encoding either a D-alanyl-D-lactate or a D-alanyl-D-serine ligase. These include vanA, vanB, vanC1, canC2, vanC3, vanD, vanE, vanG, vanL, vanM and vanN genes. Except for van $C$-type resistance, which is intrinsic to E. gallinarum and E. casseliflavus, all resistant types are acquired. ${ }^{11}$ VanA and VanB are the types most frequently detected in enterococci and have also been detected in coryneform bacteria and streptococci. ${ }^{12}$

VanA-resistant strains possess inducible, high-level resistance to vancomycin (MICs, $\geq 64 \mu \mathrm{g} / \mathrm{ml}$ ) and teicoplanin (MICs, $\geq 16 \mu \mathrm{g} / \mathrm{ml}$ ). ${ }^{13}$ Levels of vancomycin resistance among VanB isolates may range from 4 to $\geq 1000 \mu \mathrm{g} / \mathrm{ml}$ whereas susceptibility to teicoplanin is retained. ${ }^{14}$

The risk factors that have emerged for VRE infection are longer duration of hospitalization, longer stay in ICU, history of solid organ transplantation, and use of various antibiotics such as vancomycin, thirdgeneration cephalosporins, aminoglycosides, aztreonam, ciprofloxacin, imipenem, clindamycin and metronidazole. . $^{5,15,16}$

VRE infections tend to occur in more debilitated or seriously ill hospitalized patients. Mortality rates in patients with VRE bacteremia may reach 60 to $70 \%$; half these deaths may be attributable directly to the infection. However, there is no evidence that VRE are more 
virulent than vancomycin-susceptible strains of the same enterococcal species. ${ }^{5}$

Environmental surfaces and medical equipment items in the patient's room frequently become contaminated with VRE and may also serve as a reservoir for the organism in the hospital. ${ }^{17,18}$ VRE may remain viable on such surfaces for days or weeks. ${ }^{19}$

Mathematical models argue that VRE endemicity requires a constant introduction of colonized patients. Susceptibility tests that detect vancomycin resistance accurately must be used, or the prevalence of VRE may be underestimated. ${ }^{16,20}$ Several vancomycin resistance detection systems, including automated systems may misclassify isolates as intermediately susceptible to vancomycin. ${ }^{5,21}$

Despite increasing reports of VRE from different countries, there is a paucity of information on this issue from our country. ${ }^{22}$

\section{Aims and Objectives}

This study aims to identify enterococci isolated from various clinical samples up to the species level. This study also aims to determine the prevalence of vancomycin resistance among the isolates and to compare various methods of detection: disk diffusion; agar screen test - using brain heart infusion (BHI) agar containing 6 micrograms $\mu \mathrm{g}$ of vancomycin per ml; Etest (epsilometer test).

\section{Materials and Methods}

This descriptive cross-sectional study was carried out in the Department of Microbiology between June 2011 and July 2013. Permission was obtained from the Institutional Ethics Committee prior to the commencement of the study.

The data collected was entered into Microsoft Excel sheets.

Table 1: Age-wise distribution of the isolates

\begin{tabular}{|l|c|c|}
\hline & All enterococci & VRE \\
\hline Age groups (yr) & Number (\%age) & Number (\%age) \\
\hline $0-10$ & $39(25.0 \%)$ & $4(26.7 \%)$ \\
\hline $11-20$ & $16(10.3 \%)$ & $0(0 \%)$ \\
\hline $21-30$ & $26(16.7 \%)$ & $1(6.7 \%)$ \\
\hline $31-40$ & $19(12.2 \%)$ & $4(26.7 \%)$ \\
\hline $41-50$ & $15(9.6 \%)$ & $0(0 \%)$ \\
\hline $51-60$ & $17(10.9 \%)$ & $2(13.3 \%)$ \\
\hline$>61$ & $24(15.4 \%)$ & $4(26.7 \%)$ \\
\hline Total & 156 & 15 \\
\hline
\end{tabular}

Out of the 156 enterococcal isolates, $88(56.4 \%)$ were from female patients, and $68(43.6 \%)$ were from male patients. Of the 15 VRE isolates, 8 (53\%) were from female patients.

A majority of the isolates were from urine samples (61.5\%), followed by blood cultures $(18.6 \%)$ and pus samples (16\%) (Table 2). Of the 25 pus samples, 7 $(4.48 \%)$ were from burn patients (4, E. faecium; $3, E$.
All the enterococcal isolates from clinical samples such as blood, urine, pus, wound swab, cerebrospinal fluid (CSF) and other body fluids were included. All commensal enterococci from the gastrointestinal tract, female genital tract and oral cavity, and repeat isolates from the same patient were excluded.

Samples were processed according to standard procedures. ${ }^{23}$ Species-level identification was carried out as per the Facklam and Collins test scheme. ${ }^{24,25}$

All the enterococcal isolates were tested for antibiotic sensitivity against penicillin (10 U), ampicillin $(10 \mu \mathrm{g})$, chloramphenicol $(30 \mu \mathrm{g})$, erythromycin (1p5 $\mu \mathrm{g})$, ciprofloxacin $(5 \mu \mathrm{g})$, linezolid $(30 \mu \mathrm{g})$ and vancomycin $(30 \mu \mathrm{g})$ by the Kirby Bauer disk diffusion method using commercially available disks (Himedia, Mumbai, India) on Mueller-Hinton agar as per CLSI guidelines. ${ }^{26}$ HLAR was tested against gentamicin (120 $\mu \mathrm{g})$ and streptomycin $(300 \mu \mathrm{g})$. Readings of disk diffusion tests were taken at $24 \mathrm{~h}$ for vancomycin, and at $18 \mathrm{~h}$ for other antibiotics. ${ }^{26}$ Enterococci were screened for resistance using brain heart infusion (BHI) agar supplemented with $6 \mu \mathrm{g} / \mathrm{ml}$ vancomycin. ${ }^{20}$ E-tests for vancomycin and teicoplanin were performed as per manufacturer's instructions (Himedia, Mumbai, India).

Quality control of the antibiotic disks was carried out using Staphylococcus aureus ATCC 25923 and Enterococcus faecalis ATCC 29212.

\section{Results}

During the study period, 156 samples yielded isolates of enterococci. Thirty-five percent of the isolates were from the 0-20 year age group. Of the 39 patients in the 0-10 year age group, 31 were neonates, 3 of whom grew VRE. The mean age of patients with enterococcal infections was 32.1 years (Table 1). 
Twenty eight (18\%) patients presented with UTI, followed by febrile illness $(17 \%, \mathrm{n}=27)$ and septicaemia $(13 \%, \mathrm{n}=20)$. Of the patients who presented with a febrile illness, $70 \%(\mathrm{n}=19)$ had a urine culture positive for enterococcus.

Table 2: Specimen-wise distribution of the isolates

\begin{tabular}{|l|c|c|}
\hline & All enterococci & VRE \\
\hline Specimen & Number (\%age) & Number (\%age) \\
\hline Urine & $96(61.5 \%)$ & $10(66.7 \%)$ \\
\hline Blood & $29(18.6 \%)$ & $3(20.0 \%)$ \\
\hline Pus & $25(16.0 \%)$ & $2(13.3 \%)$ \\
\hline Ascitic fluid & $3(1.9 \%)$ & $0(0 \%)$ \\
\hline CSF & $2(1.3 \%)$ & $0(0 \%)$ \\
\hline ET aspirate & $1(0.64)$ & $0(0 \%)$ \\
\hline Total & 156 & 15 \\
\hline
\end{tabular}

More than half the isolates came from the medicine and paediatric wards $(36.5 \%, \mathrm{n}=57$ and $24.3 \%, \mathrm{n}=38$, respectively); 4 enterococcal isolates from each of these areas were VRE, accounting for 53\% of the VRE strains. The intensive care unit (ICU) yielded 14 strains of enterococci, of which 3 were VRE.
$135(86.5 \%)$ of the isolates were identified as $E$. faecalis, and $18(11.5 \%)$ were E. faecium. Of the remaining, two were identified as E. avium, and one as E. durans (Table 3). Thirteen (86.7\%) VRE isolates were identified as E. faecalis, and $2(13.3 \%)$ were E. faecium. The 10 remaining enterococcal isolates exhibited intermediate sensitivity to vancomycin.

Table 3: Species-wise distribution of the isolates

\begin{tabular}{|l|c|c|c|}
\hline & All enterococci & VSE & VRE \\
\hline Species & Number (\%age) & Number (\%age) & Number (\% age) \\
\hline E. faecalis & $135(86.5 \%)$ & $115(87.8 \%)$ & $13(86.7 \%)$ \\
\hline E. faecium & $18(11.5 \%)$ & $14(10.7 \%)$ & $2(13.3 \%)$ \\
\hline E. avium & $2(1.3 \%)$ & $1(0.76 \%)$ & $0(0 \%)$ \\
\hline E. durans & $1(0.6 \%)$ & $1(0.76 \%)$ & $0(0 \%)$ \\
\hline Total & 156 & 131 & 15 \\
\hline
\end{tabular}

Maximum resistance was noted to penicillin $(86.5 \%)$, erythromycin $(83.3 \%)$, and ciprofloxacin (79.5\%). $37.2 \%$ of the isolates were resistant to high level gentamicin (HL-G) $(120 \mu \mathrm{g})$, and $32.7 \%$ were resistant to high level streptomycin (HL-S) $(300 \mu \mathrm{g})$. The enterococci were least resistant to teicoplanin $(8.3 \%)$ and vancomycin $(9.6 \%)$. None of the isolates were resistant to linezolid (Table 4).

A higher proportion of resistance was noted among E. faecium strains than E. faecalis against all but two antibiotics: penicillin and ampicillin.

Table 4: Antibiotic resistance pattern of Enterococcus spp

\begin{tabular}{|l|c|c|c|}
\hline & All enterococci (n=156) & E. faecalis $(\mathrm{n}=135)$ & E. faecium $(\mathrm{n}=18)$ \\
\hline \multicolumn{1}{|c|}{ Antibiotic } & No. of R strains (\%age) & No. of R strains (\%age) & No. of R strains (\%age) \\
\hline Penicillin $(10 \mathrm{U})$ & $135(86.5 \%)$ & $117(86.7 \%)$ & $15(83.3 \%)$ \\
\hline Ampicillin $(10 \mu \mathrm{g})$ & $83(53.2 \%)$ & $72(53.3 \%)$ & $9(50.0 \%)$ \\
\hline Ciprofloxacin $(5 \mu \mathrm{g})$ & $124(79.5 \%)$ & $106(78.5 \%)$ & $16(88.9 \%)$ \\
\hline Gentamicin $(\mathrm{HL})(120 \mu \mathrm{g})$ & $58(37.2 \%)$ & $48(35.6 \%)$ & $9(50.0 \%)$ \\
\hline Streptomycin $(\mathrm{HL})(300 \mu \mathrm{g})$ & $51(32.7 \%)$ & $43(31.8 \%)$ & $6(33.3 \%)$ \\
\hline Chloramphenicol $(30 \mu \mathrm{g})$ & $92(59.0 \%)$ & $78(57.8 \%)$ & $12(66.7 \%)$ \\
\hline Erythromycin $(15 \mu \mathrm{g})$ & $130(83.3 \%)$ & $110(81.5 \%)$ & $0(0 \%)$ \\
\hline Linezolid $(30 \mu \mathrm{g})$ & $0(0 \%)$ & $0(0 \%)$ & $2(11.1 \%)$ \\
\hline Teicoplanin $(\mathrm{E}-\mathrm{test})$ & $13(8.3 \%)$ & $11(8.1 \%)$ & $4(22.2 \%)$ \\
\hline Vancomycin $(\mathrm{disk})(30 \mu \mathrm{g})$ & $19(12.2 \%)$ & $15(11.1 \%)$ & $4(22.2 \%)$ \\
\hline Vancomycin $(\mathrm{agar} \mathrm{screen})$ & $25(16.0 \%)$ & $20(14.8 \%)$ & $2(11.1 \%)$ \\
\hline Vancomycin $($ E-test) & $15(9.6 \%)$ & $13(9.6 \%)$ & No. of I strains $(\%$ age $)$ \\
\hline & $10(6.4 \%)$ & $7(5.2 \%)$ & $2(11.1 \%)$ \\
\hline Vancomycin $($ E-test) & & & \\
\hline
\end{tabular}


Table 5: Antibiotic susceptibility pattern of vancomycin-resistant enterococci

\begin{tabular}{|l|c|c|}
\hline \multicolumn{1}{|c|}{ Antibiotic } & Resistant & \%age \\
\hline Penicillin $(10 \mathrm{U})$ & 15 & $100.0 \%$ \\
\hline Ampicillin $(10 \mu \mathrm{g})$ & 12 & $80.0 \%$ \\
\hline Chloramphenicol $(30 \mu \mathrm{g})$ & 13 & $86.7 \%$ \\
\hline Erythromycin $(15 \mu \mathrm{g})$ & 13 & $86.7 \%$ \\
\hline Ciprofloxacin $(5 \mu \mathrm{g})$ & 14 & $93.3 \%$ \\
\hline Gentamicin $(\mathrm{HL})(120 \mu \mathrm{g})$ & 14 & $93.3 \%$ \\
\hline Streptomycin $(\mathrm{HL})(300 \mu \mathrm{g})$ & 12 & $80.0 \%$ \\
\hline Linezolid $(30 \mu \mathrm{g})$ & 0 & $0 \%$ \\
\hline Teicoplanin $(\mathrm{E}-\mathrm{test})$ & 13 & $86.7 \%$ \\
\hline Vancomycin $(\mathrm{E}-\mathrm{test})$ & 15 & $100.0 \%$ \\
\hline
\end{tabular}

The strains of vancomycin-resistant enterococci showed significant resistance to all the other antibiotics tested, except to linezolid. All 15 VRE strains were resistant to penicillin and $93.3 \%$ were resistant ciprofloxacin, as well as to high-level gentamicin (Table 5). A vast majority of the HLAR strains also exhibited resistance to penicillin (98\%), ampicillin (76\%), ciprofloxacin (98\%) and erythromycin (100\%).

Eleven E. faecalis strains and 2 of E. faecium exhibited VanA phenotype (vancomycin MIC $\geq 64$ $\mu \mathrm{g} / \mathrm{ml}$, teicoplanin MIC $\geq 16 \mu \mathrm{g} / \mathrm{ml})$ and 2 isolates of $E$. faecium had VanB phenotype (vancomycin MIC $\geq 64$ $\mu \mathrm{g} / \mathrm{ml}$, teicoplanin $\mathrm{MIC} \leq 8 \mu \mathrm{g} / \mathrm{ml})$.

Resistance to vancomycin was tested by 3 methods, viz. Kirby Bauer disc diffusion with vancomycin disk $(30 \mu \mathrm{g})$, BHI screen agar containing vancomycin $(6$ $\mu \mathrm{g} / \mathrm{ml}$ ), and vancomycin E-test (Table 6). The E-test showed resistance in $9.6 \%$ and intermediate sensitivity in $6.4 \%$ of the isolates.

Table 6: Vancomycin resistance detected by various methods

\begin{tabular}{|l|c|c|}
\hline & Number & \%age of enterococci \\
\hline Vancomycin disk diffusion & 19 & $12.2 \%$ \\
\hline Vancomycin screen agar & 25 & $16.0 \%$ \\
\hline Vancomycin E-test & 15 & $9.62 \%$ \\
\hline
\end{tabular}

While $79 \%$ of the strains indicated to be resistant to vancomycin by disk diffusion had a vancomycin MIC $\geq$ $32 \mu \mathrm{g} / \mathrm{ml}$ (resistant) by the E-test, only $60 \%$ of the strains that grew on vancomycin screen agar had vancomycin MIC $\geq 32 \mu \mathrm{g} / \mathrm{ml}$. Vancomycin disk diffusion had 100\% sensitivity, $97.2 \%$ specificity and vancomycin agar screen showed $100 \%$ sensitivity, $92.9 \%$ specificity.

The $E$. durans strain and one of the E. avium strains were sensitive by vancomycin disk diffusion, did not grow on the vancomycin screen agar and had vancomycin MICs $\leq 4 \mu \mathrm{g} / \mathrm{ml}$ in the E-test. The remaining $E$. avium strain exhibited intermediate sensitivity (MIC, $8-16 \mu \mathrm{g} / \mathrm{ml}$ ) to vancomycin and grew on the vancomycin screen agar, but tested sensitive to the vancomycin disk.

\section{Discussion}

Enterococci are the second most common cause of nosocomial infections in the United States, and are responsible for approximately $8 \%$ of all nosocomial bloodstream infections. ${ }^{27}$

The purpose of this study was to generate data on enterococcal infections in a rural tertiary care hospital, to identify the isolates to the species level, to assess the antibiotic resistance pattern to widely prescribed antibiotics with a focus on vancomycin resistance, and to compare the detection of vancomycin resistance by various methods.

In this study, $45(35.3 \%)$ of the enterococci were isolated from patients in the 0-20 year age group, out of which $31(19.9 \%)$ were <1 month old. The mean age of incidence of enterococcal infections was 32.13 years. This is in contrast to a studies where the average age was 62 years, and another where $96 \%$ of the patients were $>18$ years of age. ${ }^{28,29}$

The prevalence of enterococcal infections was found to be higher in female patients $(56.4 \%)$. Other studies have shown the prevalence to be $46 \%$ to $50 \%$ in females. ${ }^{28,29}$

Maximum isolates in our study were obtained from urine samples $(61.5 \%)$. Studies have reported similar observations..$^{29,30}$ In other studies, pus samples contributed the most to the number of enterococcal isolates. ${ }^{31,32}$

Urinary tract infections (UTIs) are the most common bacterial infection, and almost half of all women will experience one episode of UTI during their lifetime. ${ }^{33}$ Eighteen percent of our patients $(n=28)$ presented with UTI and additionally, 19 of patients who presented with a febrile illness grew enterococci in their urine.

We identified $135(86.53 \%)$ isolates as E. faecalis. Eighteen (11.54\%) were E. faecium, 2 (1.3\%) were $E$. 
avium and $1(0.6 \%)$ was E. durans. This is similar to studies where $87 \%$ to $90 \%$ of the strains were E. faecalis and $8 \%$ were E. faecium. ${ }^{29,30}$ Other species (E. avium, E. durans, etc.) accounted for only $2 \%$ to $3 \%$ of the isolates. ${ }^{29,30}$

The predominance of infections by E. faecalis can be related to the fact that it is found in higher numbers than other species in the faeces of most healthy adults. ${ }^{9}$ Some studies have reported a higher incidence of nonfaecalis, non-faecium species. ${ }^{34,35}$ In a Brazilian ICU, $84 \%$ of the VRE species recovered from faecal specimens of critical patients were E. gallinarum, a species with intrinsic resistance to vancomycin. ${ }^{36}$

Species identification of enterococci may be useful both as an epidemiological tool during nosocomial outbreaks and for clinical decisions about therapy. ${ }^{29}$

Of the 15 isolates of VRE, $3(20 \%)$ isolates were from patients admitted in the ICU. The infection rate by VRE in ICUs has ranged from $7 \%$ to $45 \% .{ }^{37,38}$ In India, $3.7 \%$ of the VRE isolates were from the ICU. ${ }^{39-41}$

The observed rates of penicillin $(86.5 \%)$ and ampicillin $(53.2 \%)$ resistance are comparable to other studies The rate of ciprofloxacin resistance $(79.5 \%)$ is much higher than what has been reported. ${ }^{29,35,31}$

High-level gentamicin resistance has ranged from $12.6 \%$ to $100 \%$ among E. faecalis. ${ }^{30,42}$ Thirty-seven percent of the enterococci in this study were resistant to high-level gentamicin (E. faecalis, $35.6 \%$ and $E$. faecium, $50 \%$ ). The high proportion of resistance of these HLAR strains to other classes of antibiotics is comparable to other reports. ${ }^{43}$

Vancomycin resistance among enterococci was observed to be $9.6 \%$ (E. faecalis, 9.6\%; E. faecium, $11.1 \%$ isolates). Vancomycin resistance has been observed to be on the rise: from $0.3 \%$ in $1992 ;^{29} 1.5 \%$ in $1996 ;^{32} 20 \%$ observed in $2002 ;^{40}$ to $28.6 \%$ in $2004 .^{42}$

Eleven E. faecalis strains and 2 of E. faecium exhibited VanA phenotype, and 2 isolates of E. faecium had VanB phenotype. Seventy percent of VRE in the United States exhibited the VanA phenotype, and 25\% exhibited the VanB phenotype. ${ }^{44}$ All the vancomycinresistance $E$. faecium isolates in a study from India had VanA phenotype. ${ }^{39}$ In another report, out of $5 \mathrm{VRE}$ isolates, 4 had VanA phenotype, and 1 had VanB phenotype. $^{22}$

We used three methods of antibiotic susceptibility testing to detect vancomycin resistance. Fifteen $(9.6 \%)$ strains were noted to be resistant (MIC $\geq 32 \mu \mathrm{g} / \mathrm{ml}$ ) by the E-test and 10 exhibited intermediate sensitivity (MIC 8-16 $\mu \mathrm{g} / \mathrm{ml})$. Disc diffusion indicated resistance in 19 isolates. The agar screen indicated resistance in 25 isolates.

Major errors have been reported with disc diffusion for the detection of vancomycin resistance. ${ }^{45,46}$ Similar to other reports, more than half the intermediate strains in our study were reported susceptible by disk diffusion. $^{47}$
The agar screen method for detecting VRE has been demonstrated to have $96 \%$ to $99 \%$ sensitivity and $100 \%$ specificity. ${ }^{20}$ This, however, requires careful monitoring of the vancomycin concentration in the plates. ${ }^{48} \mathrm{We}$ observed $100 \%$ sensitivity, $92.9 \%$ specificity with the agar screen; vancomycin disk diffusion had $100 \%$ sensitivity, $97.2 \%$ specificity. All the intermediate strains grew on the agar screen.

Once suspected, vancomycin resistance should be confirmed using a different method. ${ }^{16}$ Once VRE have been detected in a hospital, all enterococci should be tested for susceptibility to vancomycin. ${ }^{49}$

The E-test is a reliable susceptibility testing technique that combines the convenience of agar diskdiffusion with the precision of broth/agar dilution methods. ${ }^{47}$ Results obtained with the E-test had a high level of agreement with broth microdilution and agar dilution. ${ }^{50,51}$ This can be used as a confirmatory test for vancomycin resistance in settings that lack automated testing or molecular testing facilities.

\section{Conclusion}

Vancomycin resistant enterococci are being increasingly isolated from hospitals across the country. It is necessary to identify enterococci to the species level, as non-faecalis, non-faecium species are being increasingly isolated; some of these species are inherently resistant to vancomycin. Detection of vancomycin resistance by disk diffusion is error-prone, particularly misidentifying vancomycin-intermediate strains. Resistance may be screened for using vancomycin screen agar and should be confirmed by another method. The E-test is a suitable method for confirming resistance. Judicious use of glycopeptides is stressed on. Active surveillance for vancomycinresistant strains may be considered in endemic hospitals.

\section{References}

1. Rosenblatt-Farrell N. The landscape of antibiotic resistance. Env Health Perspect. 2009;117(6):A244-50.

2. Moellering R. Vancomycin-Resistant Enterococci. Clin Infect Dis. 1998;26:1196-9.

3. Ingerman M, Santoro J. Vancomycin: a new old agent. Infect Clin North Am. 1989;3:641-51.

4. Uttley A, Collins C, Naidoo J, George R. Vancomycinresistant enterococci. Lancet. 1988;i:57-8.

5. Cetinkaya Y, Falk P, Mayhall G. Vancomycin-Resistant Enterococci. Clin Microbiol Rev. 2000;13(4):686-707.

6. Pintado V, Cabellos C, Moreno S, Meseguer A, Ayats J, Viladrich P. Enterococcal meningitis: a clinical study of 39 cases and review of the literature. Med Balt. 2003;82(5):346-64

7. Han D, Wisniewski S, Wilson L, Barza M, Vine A, Doft $\mathrm{B}$, et al. Spectrum and susceptibilities of microbiologic isolates in the Endophalmitis Vitrectomy Study. Am J Ophthalmol. 1996;122(1):1-17.

8. Barie P, Christou N, Dellinger E, Rout W, Stone H, Waymack J. Pathogenicity of the Enterococcus in surgical infections. Ann Surg. 1990;212(2):155-9.

9. Murray B. The life and times of the Enterococcus. Clin Microbiol Rev. 1990;3(1):46-65. 
10. Hospital Infection Control Practices Advisory Committee. Recommendations for preventing the spread of vancomycin resistance: recommendation of the Hospital Infection Control Practices Advisory Committee (HICPAC). Am J Infect Control. 1995;23(2):87-94.

11. Nomura T, Tanimoto K, Shibayama K, Arakawa Y, Fujimoto S, Ike Y, et al. Identification of VanN-Type Vancomycin Resistance in an Enterococcus faecium Isolate from Chicken Meat in Japan. Antimicrob Agents Chemother. 2012;56(12):6389-92.

12. Cattoir V, Leclerq R. Twenty-five years of shared life with vancomycin-resistant enterococci: is it time to divorce? J Antimicrob Chemother. 2013;68(4):731-42.

13. Arthur M, Courvalin P. Genetics and mechanisms of glycopeptide resistance in enterococci. Antimicrob Agents Chemother. 1993;37:1563-71.

14. Quintiliani R, Courvalin P. Conjugal transfer of the vancomycin resistance determinant vanB between enterococci involves the movement of large genetic elements from chromosome to chromosome. FEMS Microbiol Lett. 1994;119:359-64.

15. Ostrowsky B, Venkataraman L, D'Agata E, Gold H, DeGirolami P, Samore M. Vancomycin-resistant enterococci in intensive care units. Arch Intern Med. 1999; 159:1467-72.

16. DeLisle S, Perl T. Vancomycin-Resistant Enterococci: A Road Map on How To Present the Emergence and Transmission of Antimicrobial Resistance. Chest. 2003;123:504S-518S.

17. Boyce J, Mermel L, Zervos M, Rice B, Potter-Bynoe G, Giorgio $\mathrm{C}$, et al. Controlling vancomycin-resistant enterococci. Infect Control Hosp Epidemiol. 1995;16:634-7.

18. Livornese L, Dias S, Samuel C, Romanowski B, Taylor $\mathrm{S}$, et al. Hospital-acquired infection with vancomycinresistant Enterococcus faecium transmitted by electronic thermometers. Ann Int Med. 1992;117:112-6.

19. Noskin G, Stosor V, Cooper I, Peterson L. Recovery of vancomycin-resistant enterococci on fingertips and environmental surfaces. Infect Control Hosp Epidemiol. 1995;16:577-81.

20. Swenson J, Clark N, Ferraro M, Sahm D, Doem G, Pfaller M, et al. Development of a standardized screening method for detection of vancomycin-resistant enterococci. J Clin Microbiol. 1994;32(7):1700-4.

21. Pendle S, Jelfs P, Olma T, Su Y, Gilroy N, Gilbert GL. Difficulties in detection and identification of Enterococcus faecium with low-level inducible resistance to vancomycin, during a hospital outbreak. Clin Microbiol Infect. 2008;14(9):853-7.

22. Mathur P, Kapil A, Chandra R, Sharma P, Das B. Antimicrobial resistance in Enterococcus faecalis at a tertiary care center of northern India. Indian J Med Res. 2003;118:25-8.

23. Forbes B, Sahm D, Weissfeld A. Overview of bacterial identification methods and strategies. In: Bailey Scotts Diagn Microbiol. 12th ed. St. Louis: Mosby; 2007. p. 216-47.

24. Facklam R, Collins M. Identification of Enterococcus Species Isolated from Human Infections by a Conventional Test Scheme. J Clin Microbiol. 1989;27(4):731-4.

25. Winn W, Allen S, Janda W, Koneman E, Procop G, Schreckenberger P, et al. Konemen's Color Atlas and Textbook of Diagnostic Microbiology. 6th ed. New York: Lipincott Williams; 2006.

26. CLSI. Performance Standards for Antimicrobial Susceptibility Testing; Twenty-First Informational
Supplement. CLSI document M100-S21. Wayne, PA: Clinical and Laboratory Standards Institute;2011.

27. Schaberg D, Culver D, Gaynes R. Major trends in the microbial etiology of nosocomial infection. Am J Med. 1991;91(Suppl 3B):72S-75S.

28. Carmeli Y, Eliopoulos G, Samore M. Antecedent Treatment with Different Antibiotic Agents as a Risk Factor for Vancomycin-Resistant Enterococcus. Emerg Infect Dis. 2002;8(8):802-7.

29. Gordon S, Swenson JM, Hill BC, Pigott NE, Facklam RR, Cooksey RC, et al. Antimicrobial susceptibility patterns of common and unusual species of enterococci causing infections in the United States. Enterococcal Study Group. J Clin Microbiol. 1992;30(9):2373-8.

30. Ruoff K, De La Maza L, Mrtagh M, Spargo J, Ferraro M. Species identities of enterococci isolated from clinical specimens. J Clin Microbiol. 1990;28(3):435-7.

31. Chaudhary U, Shamma M, Yadav A. Antimicrobial susceptibility patterns of common and unusual Enterococcus species isolated from clinical specimens. $J$ Infect Antimicrob Agents. 2007;24:55-62.

32. Vandamme P, Vercauteren E, Lammens C, Pensart N, Ieven M, Pot B, et al. Survey of enterococcal susceptibility patterns in Belgium. J Clin Microbiol. 1996;34(10):2572-6.

33. Foxman B. Epidemiology of urinary tract infections: incidence, morbidity, and economic costs. Am J Med. 2002;113(1):5-13.

34. Prakash V, Rao S, Parija S. Emergence of unusual species of enterococci causing infections, South India. BMC Infect Dis. 2005;5(14):106-10.

35. Udo EE. Species prevalence and antibacterial resistance of enterococci isolated in Kuwait hospitals. $J$ Med Microbiol. 2003 Feb 1;52(2):163-8.

36. Freitas MCS, Pacheco-Silva A, Barbosa D, Silbert S, Sader H, Sesso R, et al. Prevalence of vancomycinresistant Enterococcus fecal colonization among kidney transplant patients. BMC Infect Dis. 2006;22;6:133.

37. Bonora MG, Solbiati M, Stepan E, Zorzi A, Luzzani A, Catania MR, et al. Emergence of linezolid resistance in the vancomycin-resistant Enterococcus faecium multilocus sequence typing $\mathrm{C} 1$ epidemic lineage. J Clin Microbiol. 2006;44(3):1153-5.

38. Farr BM. For nosocomial vancomycin-resistant enterococcal infections: the ounce of prevention or the pound of cure? Clin Infect Dis. 2004;38(8):1116-8.

39. Ghoshal U, Garg A, Tiwari D, Ayyagiri A. Emerging vancomycin resistance in enterococci in India. Indian $J$ Pathol Microbiol. 2006;49(4):620-2.

40. Devi S, Rao S, Shivanand P. Characterisation, antibiotic susceptibility pattern and detection of $\beta$-lactamases in enterococci. Indian J Pathol Microbiol. 2002;45(1):7982.

41. Rahangdale V, Agrawal G, Jalgaonkar S. Study of antimicrobial resistance in enterococci. Indian J Med Microbiol. 2008;26(3):285-7.

42. Karmarkar M, Gershom E, Mehta P. Enterococcal infections with special reference to phenotypic characterization and drug resistance. Indian J Med Res. 2004;119:22-5.

43. Huidrom S, Narayanaswamy G, Dadlani R. Detection of high-level aminoglycoside resistant pattern of Enterococci isolated form urine samples at a tertiary care hospital in Bengaluru. AnnTrop Med Public Health. 2016;9:165-9.

44. Clark N, Cooksey R, Hill B, Swenson J, Tenover F. Characterization of glycopeptide-resistant enterococci 
from US hospitals. Antimicrob Agents Chemother. 1993;37:2311-7.

45. Swenson J, Hill B, Thornsberry C. Problems with the disk diffusion test for detection of vancomycin resistance in enterococci. J Clin Microbiol. 1989;27(9):2140-2.

46. Kohner PC, Patel R, Uhl JR, Garin KM, Hopkins MK, Wegener LT, et al. Comparison of agar dilution, broth microdilution, E-test, disk diffusion, and automated Vitek methods for testing susceptibilities of Enterococcus spp. to vancomycin. J Clin Microbiol. 1997;35(12):3258-63.

47. Schulz J, Sahm D. Reliability of the E test for detection of ampicillin, vancomycin, and high-level aminoglycoside resistance in Enterococcus spp. J Clin Microbiol. 1993;31(12):3336-9.

48. Hegstad K, Giske CG, Haldorsen B, Matuschek E, Schønning K, Leegaard TM, et al. Performance of the EUCAST disk diffusion method, the CLSI agar screen method, and the Vitek 2 automated antimicrobial susceptibility testing system for detection of clinical isolates of Enterococci with low- and medium-level VanB-type vancomycin resistance: a multicenter study. $J$ Clin Microbiol. 2014;52(5):1582-9.

49. Centers for Disease Control and Prevention.

Recommendations for preventing spread of vancomycin resistance. Infect Control Hosp Epidemiol. 1995;16:10513.

50. Tenover F, Swenson J, O'Hara C, Stocker S. Ability of commercial and reference antimicrobial susceptibility testing methods to detect vancomycin resistance in enterococci. J Clin Microbiol. 1995;33(6):1524-7.

51. Klare I, Fleige C, Geringer U, Witte W, Werner G. Performance of three chromogenic VRE screening agars, two Etest ${ }^{\circledR}$ vancomycin protocols, and different microdilution methods in detecting vanB genotype Enterococcus faecium with varying vancomycin MICs. Diagn Microbiol Infect Dis. 2012;74(2):171-6.

\begin{tabular}{l} 
How to cite this article: Mathew SK. A profile \\
of vancomycin-resistant enterococcal \\
infections and a comparison of resistance \\
detection methods. Indian J Microbiol Res. \\
2018;5(3):408-414. \\
\hline
\end{tabular}

